\title{
An energy efficient optimized cluster establishment methodology for sensor nodes in WSN
}

\author{
Shivshanker Biradar ${ }^{1}$, T. S. Vishwanath ${ }^{2}$ \\ ${ }^{1}$ Department of ECE, LAEC, Bidar, Karnataka, India \\ ${ }^{2}$ Department of ECE, BKIT, Bhalki, Karnataka, India
}

\begin{abstract}
Article Info
Article history:

ABSTRACT

Received Jul 14, 2020

Revised Sep 18, 2020

Accepted Nov 18, 2020

Keywords:

Base station

Cluster head

Energy efficient

Low energy adaptive clustering

hierarchy

Wireless sensor network

The compatibility of WSN is with various applications such as; healthcar eand environmental monitoring. Whereas nodes present in that network have limited 'battery-life' that cause difficulty to replace and recharge those batteries after deployment. Energy efficiency is a major problem in the present situation. In present, many algorithms based on energy efficiency have been introduced to improvise the conservation of energy in WSN. The LEACH algorithm improvises the network lifetime in comparison to direct transmission and multi-hop, but it has several limitations. The selection of CHs can be randomly done that doesn't confirm the optimal solution, proper distribution and it lacks during complete network management. The centralized EE optimized cluster establishment approach (OCEA) for sensor nodes is proposed to decrease the average energy dissipation and provide significant improvement. The proposed EE WSN model with the sensor nodes is examined under a real-time scenario and it is compared with stateof-art techniques where it balances the energy consumption of the network and decreasing the cluster head number.
\end{abstract}

This is an open access article under the CC BY-SA license.

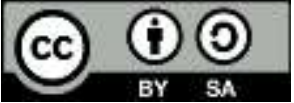

\section{Corresponding Author:}

Shivshanker Biradar

Department of Electronic and Communication Engineering

Lingaraj Appa Engineering College

Bidar, Karnataka, India

Email: shivdurga.biradar@gmail.com

\section{INTRODUCTION}

WSN is a real-time environment and it usually contains several types of user devices. The main aim of these mechanisms is to sense the data from the deployed environment and transfer towards BS [1]. WSN discover broad utilization in many fields like military applications, environmental monitoring, target tracking and structural health monitoring. WSN is composed of spatially dispersed sensors that monitor the physical parameters and environmental [2]. It interacts and gathers the data to the sink node for the analysis of the end-user. Transmitting data packets from nodes to the base station can be accomplished by various routing techniques. Because of limited nodes of power supplies, these types of components must be taken the benefits as much as they can [3]. The clustering approach comes out to be a very effective and most promising method to improve the EE [4]. Past few years, many algorithms like EE based clustering routing have been introduced to improvise the conservation of energy in WSN [3-5].

Recently, many types of research have come up with various ideas in order to minimize the energy and improvise the network longevity for proper resource utilization. In this process, the routing algorithm plays a vital role. Clustering constructs hierarchy clusters or sets of the sensing nodes, which gathers and transmits data to its $\mathrm{CH}$. The $\mathrm{CH}$ groups the data and transmits to BS/sink that acts as middleware among 
network and end-user. LEACH and clustering algorithm are the classical protocol that considers the energy for hierarchical data routing [6]. The network can be grouped onto the clusters and SN (sensor node) which sends their data to corresponding $\mathrm{CH}$. The protocol randomly chooses $\mathrm{CHs}$ in a stochastic manner for each round. $\mathrm{CH}$ interacts with every single cluster node which is known as the $\mathrm{SN}$ to sense and collect the data.

$\mathrm{CH}$ allocates schedules of time division multiple access (TDMA) to its corresponding cluster member $(\mathrm{CM})$. The member node can transfer the data during the allocated time-slot. The data can be checked for the redundancy and compacted before interacting with the sink-node. The CHS directly interacts with BS in the protocol of LEACH; hence the consumption of power is transmitting the data from $\mathrm{CH}$ to BS that will be compared to communication among CHs. As an outcome, $\mathrm{CHs}$ exhausted its energy within a short time. On the other side, in order to overcome this issue, multi-hop communication can be very helpful, but still, it is not effective in small networks.

Selecting $\mathrm{CH}$ is the sophisticated job as different factors that have to be assumed for the best selection node in a cluster [7]. These different factors consist of distance among mobility, throughput, residual energy, and nodes of every single node. The algorithm of LEACH improvises the network lifetime in comparison to multi-hop and direct transmission but still, it has several limitations. The selection of CHs can be randomly done that doesn't confirm the optimal solution and proper distribution. The nodes with less energy have the same priority as with high energy levels to be selected as the $\mathrm{CH}$. While, lower residual energy node achieves chosen to serve as the $\mathrm{CH}$, and quickly expires the resulting in a short network span [8].

LEACH-ME [9] chooses the SN with a lower mobility factor to take benefits on the role of $\mathrm{CH}$. The CBR (cluster-based routing) protocol for MNs (mobile nodes) [10] uses the design of a cross-layer to manage mobility. The MBC (mobility based clustering) [11] protocol selects SN to $\mathrm{CH}$ on the basis of its remaining mobility and energy. The main drawback of this protocol is the threshold function that choosing the SN to be $\mathrm{CH}$ that may be very smaller than the LEACH, because it is multiplied simultaneously by the mobility factor and energy factor that leads to unbalance $\mathrm{CHs}$ number, thereby maximizing the dissipation of energy. Moreover, LEACH-ME [12] utilizes the fuzzy logic to combine more than one factor for the selection of CH. In this paper, the centralized EE clustering routing protocol for sensor nodes is introduced to reduce the average dissipation of energy and provide promising improvement. The main contribution of this work: the optimized Clustering algorithm is improved that periodically chooses the optimal set of the CHs according to an average node of hybrid energy and average speed of node. This is crucially eminent that it utilizes the distributed clustering protocol in order to choose the $\mathrm{CHs}$ on the basis of current node speed and residual node energy.

\section{LITERATURE SURVEY}

In the past few decades, several studies have been devoted to optimizing the cluster size in the WSNs. The main idea of dynamic clustering is introduced HEED [13], LEACH [14] aims to improvise EE. Anyways, performing the dynamic clustering executes more overhead on the network. This problem has been not solved in the model of unequal-clustering-size (UCS) [15] as the first scheme of unequal clustering. The main target of schemes $[16,17]$ is to address the problem oh hot-spot that could happen in the clusters around BS since these types of clusters need extra power to relay data-packets from the outer clusters. In UCS, the CHs consumption of balanced energy can be improved by adjusting the number of nodes in various clusters based on traffic load.

Thus, UCS improvise EE that outcomes in the lifetime of a longer network. Additionally, utilizing a 2-hop of inter-cluster communication that leads to reduce the transmission distance in comparison to the LEAH. Anyways, the network is parted into 2 layers whereas UCS is inapplicable for the larger size of networks. Furthermore, the energy consumption of inter-cluster is not assumed in the UCS. The randomization technique can be distributed energy between SNs in the network. LEACH $[18,19]$ considers each node that has the radio powerful to reach directly to the sink node. But operating radio in its maximum power that depletes the battery rapidly.

Additionally, it can be observed that the consumption of balanced energy is accomplished by assigning the larger size of the cluster to $\mathrm{CHs}$ that belonging to the outer layers. It has very little communication [20], whereas this problem is not considered in the energy-balanced-data-gathering (EBDG) [21]. To improve the lifetime of the network, the coverage time optimization-based method is represented [22] that intends to compute the optimal size of the cluster in various layers. Anyways, one of the main desires of such a method is several clusters; however, it is not feasible [23]. Arranging the ACT (Transmission ranges) and cluster sizes methods have been represented by [23] that intends to overcome the problem of a hot spot and improve the network lifetime. In the ACT, the network topology contains many layers and cluster size that belonging to every layer is defined according to distance from the BS. Therefore, 
this property can resolve the problem of an energy hole. Anyways, clusters size fitting is very larger to the outermost clusters that lead to improvising the energy consumption of intra-cluster as outer layers. Additionally, these new locations are chosen as the $\mathrm{CH}$ deviates from ideal ones and this property creates very energy distribution.

In [24] park method defines $\mathrm{CH}$ selection based on the K-Means algorithm. In the network, the SNs are gathered into the clusters. Before cluster formation, each $\mathrm{CH}$ is chosen randomly based on the minimum distance of Euclidean among MNs and centroid points. The main problem with the park method is the time that takes for the formation of a cluster. The EBCAG based scheme (energy-balancing-unequal-clusteringapproach for gradient) is represented by [25]. The EBCAG intends to improvise the size of the cluster in various network layers to avoid unbalanced nodes of energy consumption that belonging to the various layers. The cluster size is computed by the help of gradient values of CHs that are calculated based on received data from given nodes with the gradient values. This method balances the CHs power consumption as well as it disables the disadvantages of optimization-based cluster size methods.

Anyways, EBCAG creates a smaller size of the cluster and it is the opposite of being the network scalability. In DBS [26], the weal point has been removed by giving the mathematical framework to improvise the size of the cluster in various network layers. The DBS distributes network into the coronas. After that, various clustering methods are implemented in every single corona that outcomes in improved energy conservation and LB (load balancing). The algorithm of sub-clustering named OCCN (optimalclustering in circular-networks) is introduced by [27] to improve the lifetime of network and minimize dissipated energy. In the end, consumption of energy can be improved by diving the network into the same size of clusters in a distributed manner. Anyways, the same size of cluster leads to a difference in the consumption of energy during inter-cluster communication that outcomes at initial network partitioning.

\section{RESEARCH METHOD}

A WSN model with the mobile nodes (MNs) is examined in this paper. It is assumed that this model of the network consists MNs that uniformly scattered in the area $(\mathrm{z} \times \mathrm{z}$ meters) and WSN clustered diagram is shown in Figure 1. The assumptions of the network model are given below:

a) The BS is fixed and located in a sensing field sensor.

b) The FNs and BS are nor doing the energy constrain, while the MNs are the energy-constrained.

c) Each node knows its velocity, current position, and energy level.

d) Each node can use the power setting to change the number of transmitted power via RSSI (ReceivedSignal-Strength-Indication).

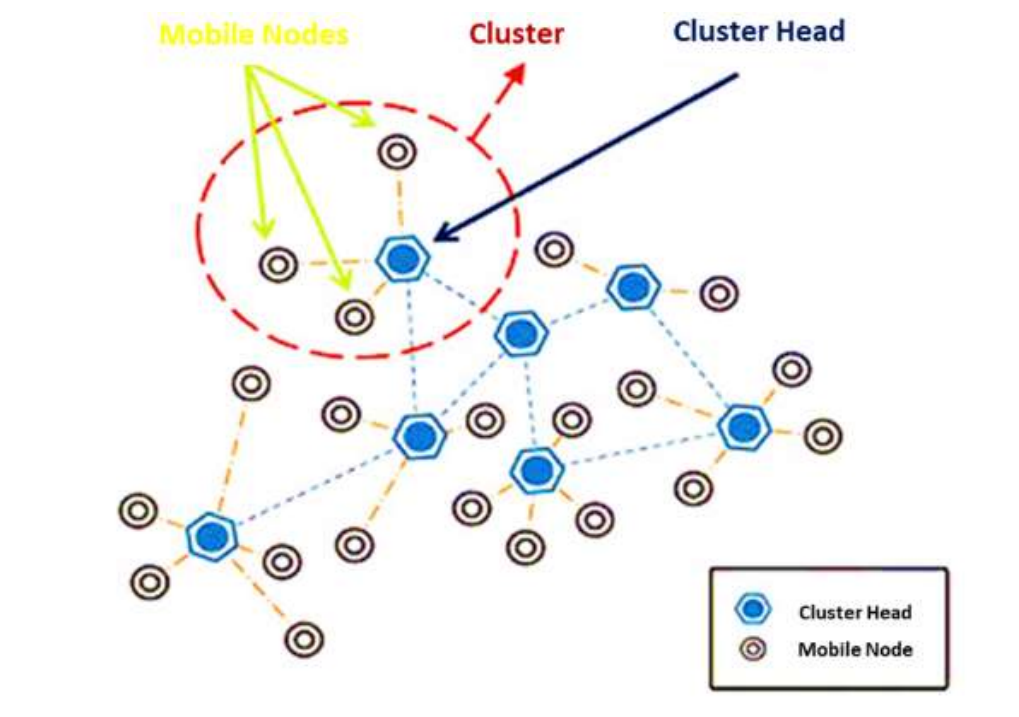

Figure 1. WSN Clustered Diagram

These above-given assumptions as reasonable for several real networks. Such as FNs, can be powered by the help of energy harvesting and have a continuous supply of power [28]. In this case, each 
node knows its current speed and position that can be obtained by location and GPS algorithms [29, 30]. Similarly, each node can evaluate its level; of energy according to specific hardware of the energy model.

The MNs energy consumption is measured by similar model as in [31], where the $a$ distance between the receiver and transmitter is considered. If $a$ distance is lesser to considered $a_{0}$ threshold then the open space channel-model is considered with energy loss $(\vartheta)$ or else the model of multipath channel $(\mu)$ is considered. So in order to send and receive $b$ number of bit message signal at $a$ distance, the cost of transmitting and receiving are given as;

$$
D_{T}(b, a)=b D_{e d}+b c_{s c} \vartheta
$$

Where $a$ distance is lesser then $a_{0}$

$$
\text { D_T }(b, a)=b \text { D_ed+bc_mp } \mu
$$

Where $a$ distance is greater or equal to $a_{0}$

$$
D_{R}(l)=b D_{e d}
$$

Here, $D_{e d}$ denotes for energy dissipation/bit at process of transmitting and receiving, where $c_{s c}$ denotes for parameter of amplify energy and corresponds to open space channel-model. Moreover, $c_{m p}$ is a parameter of amplify energy and corresponds to multipath channel-model.

All MNs from a network is given by $E=\left\{e_{1}, e_{2}, e_{3}, \cdots, e_{n}\right\}, g$ denotes the time scale of polling $(g=$ $01,2, \cdots)$. Starting phase, a round $g$ for each $e_{i}$ transmit message towards the base station, where $i$ values ranges from 1 to $n$. However, the transmitted message contains a node location, $H\left(e_{i}\right)$ present node speed and $D\left(e_{i}\right)$ energy level of node.

In accordance to this transmitted message, initially base station computes the average of node energy follow as;

$$
\bar{D}_{n}=\frac{1}{n} \sum_{i=1}^{n} D\left(e_{i}\right)
$$

Also compute the average of node speed follow as;

$$
\bar{H}_{n}=\frac{1}{n} \sum_{i=1}^{n} H\left(e_{i}\right)
$$

Where, $e_{i} \in E$. The base station chooses a number of nodes $K$ from $E$ set $(K \subseteq E)$ and given as;

$$
K=\left\{\begin{array}{l}
K_{1} \cap K_{2}, \text { if }\left|K_{1} \cap K_{2}\right| \geq L_{b} \\
K_{1} \cup K_{2}, \text { if }\left|K_{1} \cap K_{2}\right|<L_{b}
\end{array}\right.
$$

The symbol of $L_{b}$ denotes for optimal channel heads (CHs) number [31], the $K_{1}$ and $K_{2}$ shows set of nodes, and $\left|K_{1} \cap K_{2}\right|$ shows the cardinality of $K_{1} \cap K_{2}$. Moreover, energy level of nodes is above then the threshold/average energy value, and the computed current speeds below then the value of average speed is;

$$
\begin{aligned}
& K_{1}=e_{j} \mid D\left(e_{j}\right) \geq \bar{D}_{n}, \forall e_{j} \in E \\
& K_{2}=e_{j} \mid H\left(e_{j}\right) \geq H_{n}, \forall e_{j} \in E
\end{aligned}
$$

While considering a condition where $\left|K_{1} \cap K_{2}\right| \geq L_{b}$, this implies that set of nodes in $K$ are having low speeds and high energy levels are qualified to become $\mathrm{CHs}$ during that particular round. Whereas considering a condition $\left|K_{1} \cap K_{2}\right|<L_{b}$, this implies that set of nodes in $K$ are having low speeds or high energy levels are probable to become $\mathrm{CHs}$ during that particular round.

The set of nodes from $K$ is considered to perform an optimization process to get optimal $\mathrm{CHs}$ that becomes $\mathrm{CHs}$ at a particular round. Here several processing levels is used to get optimal $\mathrm{CHs}$ such as; initialization level, neighborhood finding level and final/completion level.

At initialization level, initially stochastically set of $\mathrm{CHs}$ is generated and denoted by $L=$ $\left\{l_{1}, l_{2}, l_{3}, \cdots\right\}$ from $K$ and $m=0$ in order to ensure $|L|=L_{b}$. At neighborhood finding level, $m=m+1$ is considered to find out a novel set of nodes $N$, which shows random perturbation of $L$ nodes. Therefore, the new $\tilde{\alpha}$ and $\tilde{\beta}$ coordinates of nodes in $N$ are computed through $\alpha$ and $\beta$ coordinates of nodes in $L$. 


$$
\begin{aligned}
& \tilde{\alpha}=\alpha+\operatorname{rand}\left(-c_{\text {max }}, c_{\text {max }}\right) \\
& \tilde{\beta}=\beta+\operatorname{rand}\left(-c_{\text {max }}, c_{\text {max }}\right)
\end{aligned}
$$

Where, rand function is considered to generate the random numbers and $c_{\max }$ denotes the maximal change at current random perturbation.

Afterwards, it search the neighborhood nodes in $K$ which has located near to $(\tilde{\alpha}, \tilde{\beta})$, so on these nodes selects a novel set of CHs and denoted by $\hat{L}$ (this can be infer as $L_{b}=|L|=|N|=|\hat{L}|$ ). In present state is presented through $L$ set of $\mathrm{CHs}$ with $f(L)$ cost function and its neighborhood state is presented through $\widehat{L}$ set of $\mathrm{CHs}$ with $f(\widehat{L})$ cost function.

At considered $m$ iteration, the present state $L$ will change to $\hat{L}$ neighborhood state and the probability can be computed as follows;

$$
N(L \rightarrow \hat{L})=\exp \left[-f(\hat{L})-f(L) / Q_{m}\right]
$$

The above given equation (11) is true when $f(\hat{L}) \geq f(L)$, otherwise the probability of $L \rightarrow \hat{L}$ will be one. Where, $Q_{m}$ denotes for control factor parameter and given by;

$$
Q_{m}=R_{h p} \times e^{-\frac{m}{20}}
$$

Where, $R_{h p}$ is hyper parameter value that ranging from 100 to 10000 . The computed value of $Q_{m}$ help to decide the good clusters and function $f($.$) signifies the cost function as;$

$$
f(L)=\sum_{i=1}^{n} \min _{i} \in E, l_{i} \in L \quad \vartheta\left(e_{i}, l_{i}\right)
$$

Where, distance in between the $e_{i}$ node and $l_{i}$ channel head is given by $a($.$) . If L$ present state become $\hat{L}$ neighborhood state then $L=\hat{L}$.

In final level, $S_{i t r}$ stands for considered total iterations, where $S_{i t r}$ is greater or equal to $m$ then algorithm is terminated otherwise it again go to neighborhood finding level. Once the determination of optimal CHs are done, the base station used to broadcast the message which consist of CH's ID for every node present in a network. However, a particular node ID same to CH's ID then it became a CH. Else the node selects its slot of TDMA for process of 'data-transfer' and node falls dead till the time has come to forward signal to its $\mathrm{CH}$.

\section{RESULTS AND DISCUSSION}

In this section, the authors provided result and simulation analysis of our proposed OCEA with respect to other state-of-art technologies. The simulation is carried out in Matlab-2016b with system configuration of 12GB RAM, Intel i5 processor and latest Windows operating system. A WSN model with the sensor nodes is examined and it is assumed that our network model consists of 200 number of sensor nodes that uniformly scattered in the $100 \times 100$-meter monitoring area. The assumptions of the network model have been made where; the FNs and BS are nor doing the energy constrain, while the MNs are the energy-constrained. Moreover, each node knows its velocity, current position, and energy level and considered assumptions are reasonable for several real networks.

It is known that LEACH approach saves the energy of nodes in order to optimize efficiency of network in some aspects, but it lacks during complete network management. In addition, it is not much efficient to balance the energy consumption of network and to select the cluster heads sum. The OCEA algorithm periodically chooses the optimal set of the $\mathrm{CHs}$ according to an average node of hybrid energy and average speed of node.

To compare with our proposed approach, energy balance clustering routing algorithm (EB-CRA) is considered [32]. In [32], they randomly scattered 200 nodes in the $100 \mathrm{~m} \times 100 \mathrm{~m}$ area, which is similar as per our simulation initialization. The EB-CRA is proposed for cluster head selection and BS communication, it considers the node residual energy and distance between the BS and $\mathrm{CH}$. Therefore, the possibility of node energy is to be designated as the $\mathrm{CH}$ and the $\mathrm{CH}$ is used at the process of data transmission due to more distance from the BS. Moreover, the ACO algorithm [32] has perform better than the LEACH algorithm, so the authors consider this approach for $\mathrm{CH}$ energy consumption comparison. 
Figure 2 shows the optimized cluster establishment and localization of $\mathrm{CHs,} \mathrm{where} \mathrm{(a)} \mathrm{represents} \mathrm{at}$ 1 poll, (b) represents at 10 poll and (c) represents at 500 poll. It is clearly noted that while increase in polling number or iteration or round, the number of clusters has increased but after several polling round it is not increasing. Which states that it has achieved is optimal cluster number. Increasing in number of polling, the localization of $\mathrm{CHs}$ is going to better and better.

$\mathrm{CH}$ nodes energy consumptions is very essential indicator to evaluate network efficiency and performance, Figure 3 shows energy consumption (Joules) of $\mathrm{CH}$ as per polling number. It shows that as per increment of polling, the energy consumption $\mathrm{CHs}$ in a network is decreasing. In case, polling round delay time is more compared to threshold value then the $\mathrm{SN}$ is considered to be dead node, Figure 4 represents the number of dead nodes as per polling number. Where it seems that till 160 polling number the dead nodes are zero and afterwards observed linear pattern between number of dead nodes (i.e., ranging from 0 to 60) and polling number (i.e., ranging from 160 to 500).

Figure 5 shows the average number of $\mathrm{CHs}$ as per polling number, this computed by taking mean CHs at each 100 polls. In Figure 5, from polls 1 to 100 and 101 to 200, the average number of CHs is same 37. While from 201 to 300,301 to 400 and 401 to 500, the average number of CHs are 33, 31, and 27. It indicates the average of $\mathrm{CHs}$ count is decreasing as per increasing in number of polls.

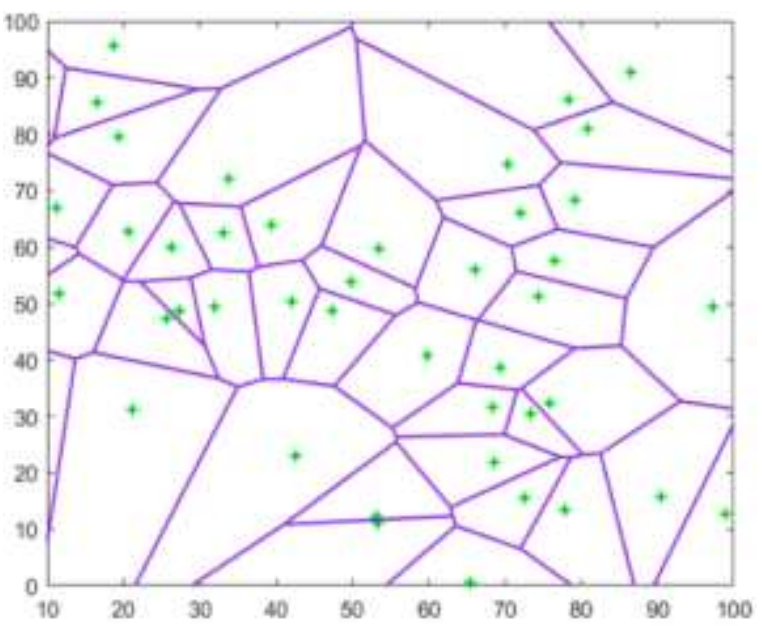

(a)

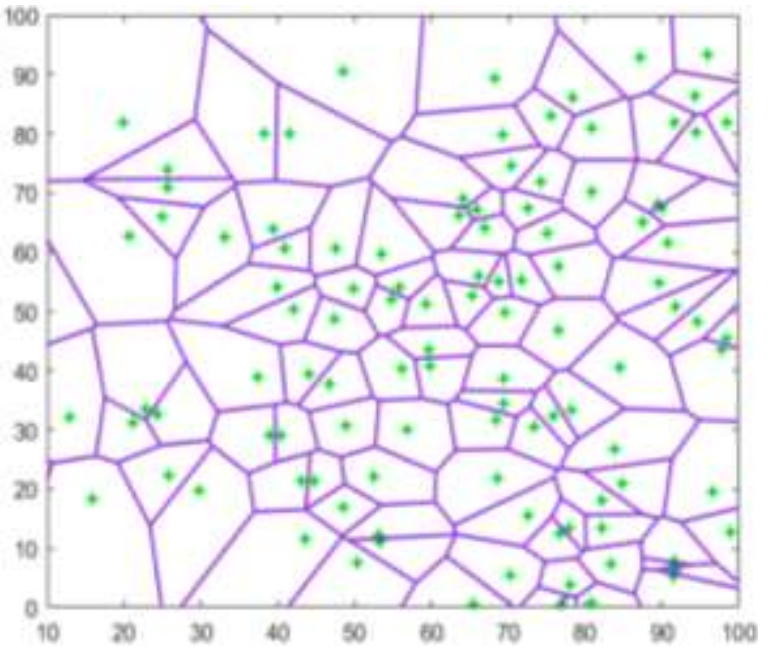

(c)

Figure 2. Cluster establishment and Localization of CHs; (a) 1 poll, (b) 10 poll and (c) 500 poll

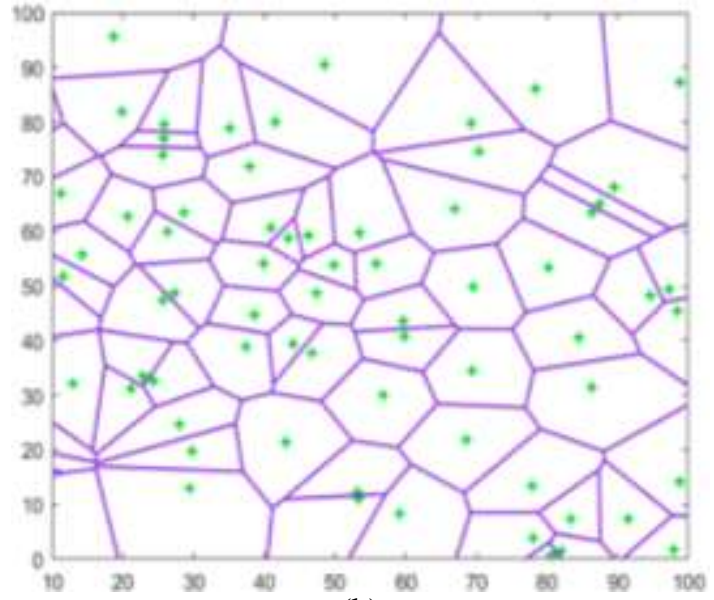

(b)

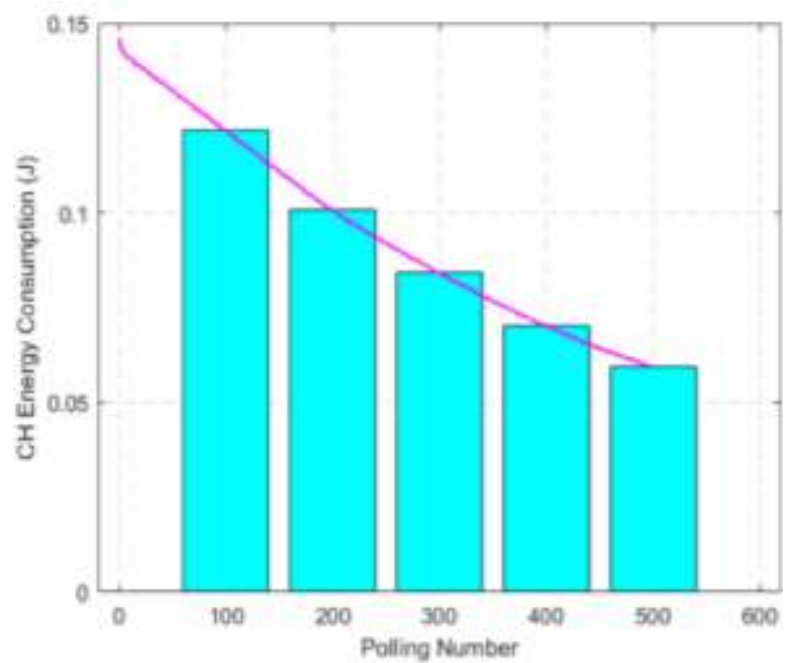

Figure 3. Energy consumption of $\mathrm{CH}$ as per polling number 


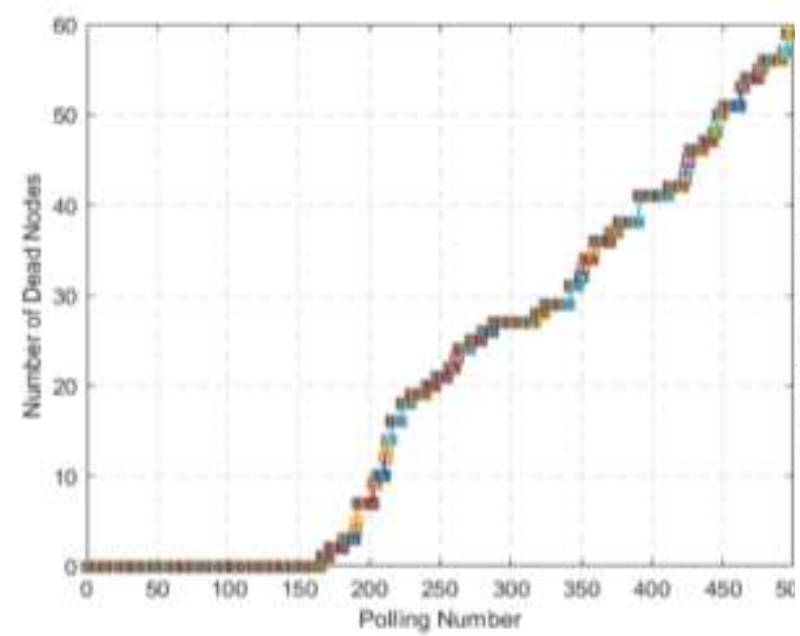

Figure 4. Number of dead nodes as per polling number

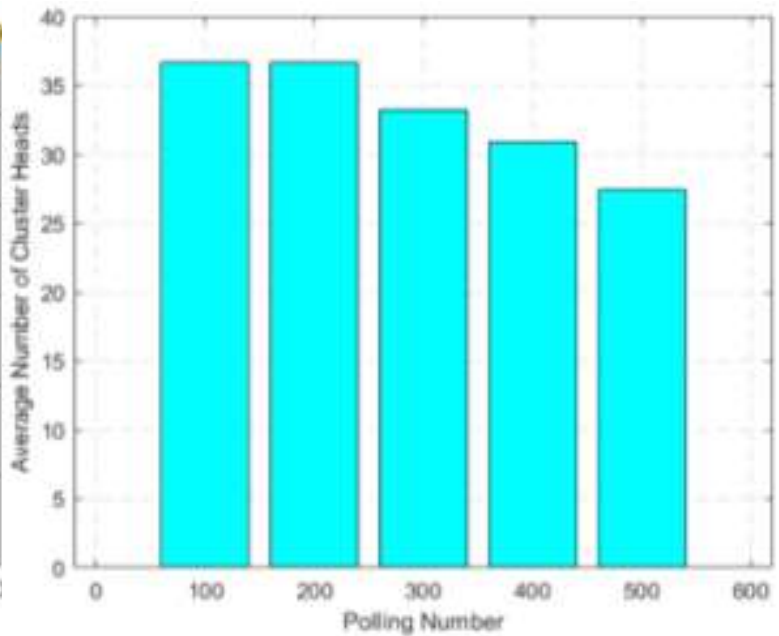

Figure 5. Average number of $\mathrm{CHs}$ as per polling number

Table 1 shows the energy comparison at different number of polls and same thing represented in a graphical format in Figure 6, where it considered different poll number to compare energy consumptions. At 100 poll number, our proposed methodology has got $11.35 \%, 64.7 \%$ and $45.8 \%$ less energy consumption compared to EB-CRA, LEACH and ACO techniques. Similarly, at 500 poll number, our proposed methodology has got $50.8 \%, 75.68 \%$ and $65.11 \%$ less energy consumption compared to EB-CRA, LEACH and ACO techniques. It indicates that difference in energy consumption has increased more with increment in polling number and our proposed methodology shows ability to manage less energy consumption at $\mathrm{CHs}$.

Table1. Energy comparison at different number of polls

\begin{tabular}{ccccc}
\hline No. of Polls & ACO & LEACH & EB-CRA & OCEA \\
\hline 100 & 0.228 & 0.3501 & 0.1394 & 0.123576 \\
200 & 0.2709 & 0.3 & 0.1141 & 0.104796 \\
300 & 0.2 & 0.2649 & 0.0791 & 0.087338 \\
400 & 0.1723 & 0.3248 & 0.1376 & 0.072483 \\
500 & 0.175 & 0.2511 & 0.1243 & 0.061048 \\
\hline
\end{tabular}

\section{Energy Comparision Chart}

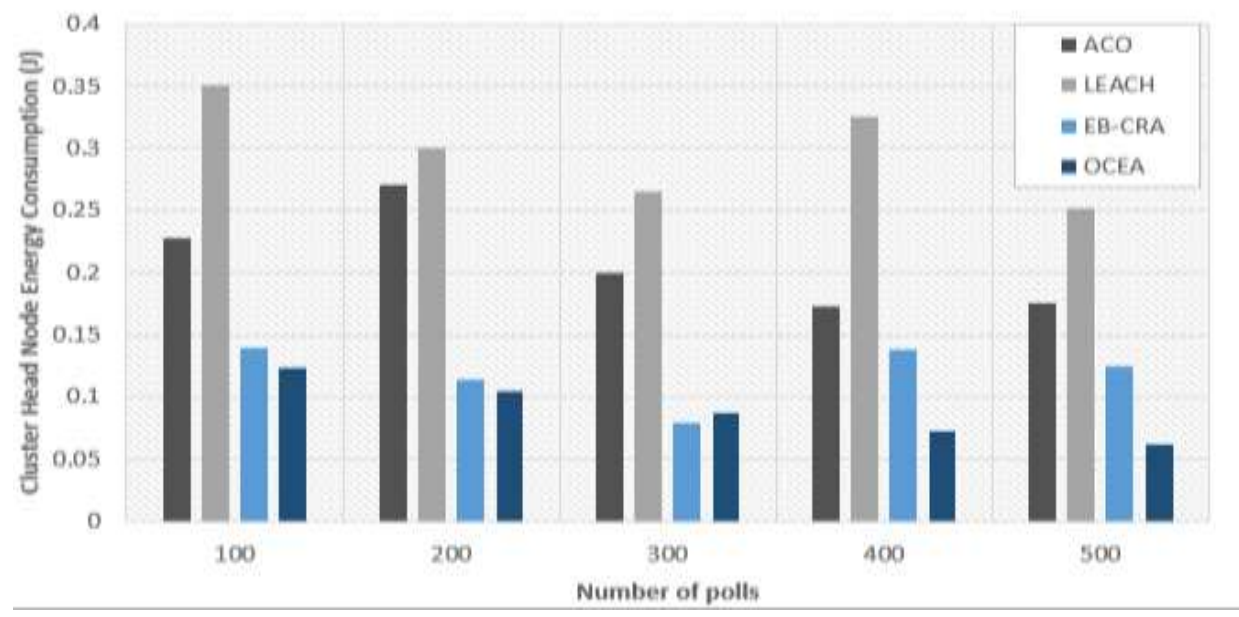

Figure 6. Graphical representation of energy comparison at different number of polls 


\section{CONCLUSION}

In order to improve the lifetime and efficiency of the network, the computation of optimal cluster and localization of $\mathrm{CHs}$ is very important. Therefore in this study the authors proposed OCEA, which periodically chooses the optimal set of the CHs according to an average node of hybrid energy and average speed of node. In the result analysis section, shows the localization CHs in various polls, energy consumption with respect to polling round has also shown. It analyzed that $\mathrm{CH}$ nodes energy consumptions is decreasing as per polling round, which very essential indicator to evaluate network efficiency and performance. To compare with our proposed approach, EB-CRA, ACO and very popular LEACH protocol is considered in the validation part, where $\mathrm{CH}$ energy consumption is compared at various polls. Whereas, OCEA approach shows much effectiveness to balance the energy consumption of network and to select the cluster heads sum.

\section{REFERENCES}

[1] Magadevi, N., and Kumar, V. J. S. "Energy efficient, obstacle avoidance path planning trajectory for localization in wireless sensor network," Cluster Computing, pp. 1-7, 2017.

[2] Akyildiz, I. F., Su, W., and Cayirci, E., "A survey on sensor networks," IEEE Communications Magazine, vol. 40, no. 8, pp. 102-114, 2002.

[3] Gharaei, N., Bakar, S. Z. M., and Pourasl, A. H., "Inter-and intra-cluster movement of mobile sink algorithms for clusterbased networks to enhance the network lifetime," Ad Hoc Networks, vol. 85, pp. 60-70, 2019.

[4] Dagdeviren, O., and Erciyes, K, "Graph matching-based distributed clustering and backbone formation algorithms for sensor networks," The Computer Journal, vol. 53, no. 10, pp. 1553-1575, 2010.

[5] Tatsis M., Skoutas N, Vouyioukas D., and Skianis C, "Energy-aware clustering of CoMP-DPS transmission points," Computer Communications, vol. 135, pp. 28-39, 2019.

[6] W. R. Heinzelman, A. Chandrakasan, and H. Balakrishnan, "Energyefficient communication protocol for wireless microsensor networks," in Proceedings of the 33rd Annual Hawaii International Conference on System Sciences, 2000, p. 10.

[7] M. Chatterjee, S. K. Das, and D. Turgut, "An on-demand weighted clustering algorithm (WCA) for ad hoc networks," in Global Telecommunications Conference, vol. 3, pp. 1697-1701, 2000.

[8] J. Xu, N. Jin, X. Lou, T. Peng, Q. Zhou, and Y. Chen, "Improvement of LEACH protocol for WSN," in Fuzzy Systems and Knowledge Discovery (FSKD), 2012 9th International Conference on, 2012, pp. 2174-2177.

[9] G. S. Kumar, P. M. V. Vinu, and K. P. Jacob, "Mobility metric based LEACH-Mobile protocol," in Proc. 16th Int. Conference Advance Comp. Comm. (ADCOM), Dec-2008, pp. 248-253.

[10] C. K. Ng, N. K. Noordin, and M. F. A. Rasid, "Cluster based routing protocol for mobile nodes in wireless sensor network," Wireless Comm., vol. 61, no. 2, pp. 251-281, Nov. 2011.

[11] S. Deng, J. Li, and L. Shen, "Mobility-based clustering protocol for wireless sensor networks with mobile nodes," IET Wireless Sensor Syst., vol. 1, no. 1, pp. 39-47, Mar. 2011.

[12] J.S. Lee and C.L. Teng, "An enhanced hierarchical clustering approach for mobile sensor networks using fuzzy inference systems," IEEE IoT., Aug. 2017, vol. 4, no. 4, pp. 1095-1103.

[13] Younis, O. and S. Fahmy, "HEED: A hybrid, energy-efficient, distributed clustering approach for ad hoc sensor networks," IEEE Transactions on mobile computing, vol. 3, no. 4, pp. 366-379, 2004.

[14] Heinzelman, W. R., A. Chandrakasan and H. Balakrishnan, "Energy-efficient communication protocol for wireless microsensor networks," Proceedings of the 33rd annual Hawaii international conference on IEEE, System sciences, 2000.

[15] Soro, S. and W. B. Heinzelman, "Prolonging the lifetime of wireless sensor networks via unequal clustering," Parallel and Distributed Processing Symposium, Proceedings 19th IEEE International. 2005.

[16] Bagci, H. and A. Yazici, "An energy aware fuzzy approach to unequal clustering in wireless sensor networks," Applied Soft Computing, vol. 13, no. 4, pp. 1741-1749, 2013.

[17] Selvi, G. V. and R. Manoharan, "Balanced unequal clustering algorithm for wireless sensor network," I-Manager's Journal on Wireless Communication Networks, vol. 3, no. 4, pp. 23, 2015.

[18] Agarwal, K., and Muruganandam, K. "Low energy adaptive clustering hierarchy (leach) protocol: Simulation and analysis using matlab," Power and communication technologies-GUCON, 2018, pp. 60-64.

[19] Behera, T. M., and Mohapatra, S. K., "Energy-efficient modified LEACH protocol for IoT application," IET Wireless Sensor Systems, vol. 8, no. 5, pp. 223-228, 2018.

[20] Shu, T. and M. Krunz "Coverage-time optimization for clustered wireless sensor networks: a power-balancing approach,” IEEE/ACM Transactions on Networking, vol. 18, no. 1, pp. 202-215, 2010.

[21] Zhang, H. and H. Shen, "Balancing energy consumption to maximize network lifetime in data-gathering sensor networks," IEEE Transactions on Parallel and Distributed Systems, vol. 20, no. 10, pp. 1526-1539, 2009.

[22] Moon, S.-H. and S.-J. Han., "Lifetime optimization for large-scale sink-centric Wireless sensor networks," Modeling and Optimization in Mobile, Ad Hoc, and Wireless Networks (WiOpt), IEEE. 2015, pp. 213-220.

[23] Lai, W. K., and L. Y. Lin, "Arranging cluster sizes and transmission ranges for wireless sensor networks," Information Sciences, vol. 183, no. 1, pp. 117-131, 2012.

[24] Park, G. Y., Kim, H, and Youn, H. Y., "A novel cluster head selection method based on k-means algorithm for energy efficient wireless sensor network" Advanced Information Networking and Applications Workshops (WAINA), IEEE 2013, pp. 910-915. 
[25] Liu, T., and P. Liang "An energy-balancing clustering approach for gradient-based routing in wireless sensor networks," Computer Communications, vol. 35, no. 17, pp. 2150-2161, 2012.

[26] Amini, N., W. Xu, and M. Sarrafzadeh, "Cluster size optimization in sensor networks with decentralized clusterbased protocols," Computer communications, vol. 35, no. 2, pp. 207-220, 2012.

[27] Arghavani, M., M. Esmaeili, and A. Arghavani, "Optimal energy aware clustering in circular wireless sensor networks," Ad Hoc Networks, vol. 65, pp. 91-98. 2017.

[28] F. K. Shaikh and S. Zeadally, "Energy harvesting in wireless sensor networks: A comprehensive review," Renewable-Sustain-Energy, vol. 55, pp. 1041-1054, Mar. 2016.

[29] L. Karim, N. Nasser, and T. E. Salti, "RELMA: A range free localization approach using mobile anchor node for wireless sensor networks," in Proc. IEEE Global Telecommun. Conf., Dec. 2010, pp. 6-10.

[30] Y. Xu, J. Heidemann, and D. Estrin, "Geography-informed energy conservation for ad hoc routing," in Proc. of Mobile Computer Network. (MOBICOM), Jul. 2001, pp. 70-84.

[31] W. B. Heinzelman, A. and H. Balakrishnan, "An application-specific protocol architecture for wireless microsensor networks," IEEE Transaction. wireless Communication., vol. 1, no. 4, pp. 660-670, Oct. 2002.

[32] Y. Xu, Z. Yue and L. Lv, "Clustering routing algorithm and simulation of internet of things perception layer based on energy balance," in IEEE Access, vol. 7, pp. 145667-145676, 2019.

\section{BIOGRAPHIES OF AUTHORS}

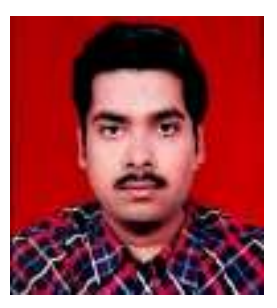

Shivshanker. P. Biradar. Completed B.E (Electronics and Communication engineering), M. Tech (Communication System) and perusing Ph.D from VTU. Research work at congestion control in sensor network using routing techniques. Interest of area wireless communication, digital processing etc.

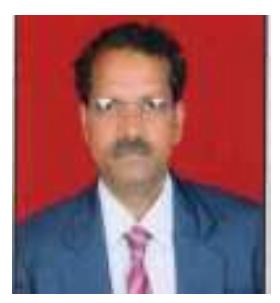

T.S.Vishwanath Ph.D in Control System, Institution: Dr MGR Educational and Research Institute University Chennai India, M. E. in Control System, Institution: WCE Sangli, BE in Electrical Engineering, Institution: PDA College of Engineering Gulbarga. Research work at Robust $\mathrm{H} \infty$ control and analysis" application to electrical drives. Member of Institute of Engneer (MIE), ISTE, IETE and IJEET. 